\title{
Melatonin prevents sepsis-induced renal injury via the PINK1/Parkin1 signaling pathway
}

\author{
WENLING DAI $^{1 *}$, HAIFENG HUANG ${ }^{2 *}$, LINJIE SI $^{1}, \mathrm{SHI} \mathrm{HU}^{1}$, \\ LIANGLIANG ZHOU ${ }^{1}$, LINGLING XU ${ }^{3}$ and YIJUN DENG ${ }^{1}$ \\ ${ }^{1}$ Department of Critical Care Medicine, Yancheng City No. 1 People's Hospital, Yancheng, Jiangsu 224006; \\ ${ }^{2}$ Department of Clinical Laboratory, Sheyang Zhenyang Hospital, Yancheng, Jiangsu 224300; \\ ${ }^{3}$ Department of Clinical Laboratory, Yancheng City No. 1 People's Hospital, Yancheng, Jiangsu 224006, P.R. China
}

Received March 8, 2018; Accepted September 4, 2018

DOI: $10.3892 /$ ijmm.2019.4306

\begin{abstract}
Melatonin(N-acetyl-5-methoxytryptamine; MT) has been shown to have a protective effect against sepsis-induced renal injury, however, the mechanisms underlying the function of MT remain to be elucidated. Therefore, in the present study, the potential mechanisms underlying the preventive role of MT in sepsis-induced renal injury were investigated. Hematoxylin and eosin staining was used to detect the effect of MT on the reduction of renal tissue damage, and immunohistochemistry (IHC), ELISA and western blot analysis were performed to determine the influence of MT on the protein expression of PTEN-induced putative kinase 1 (PINK1), nucleotide-oligomerization binding domain and leucine-rich repeat pyrin domain-containing 3 (NLRP3), apoptosis-associated speck-like protein containing a C-terminal caspase recruitment domain (ASC1), interleukin (IL)-18, IL-1 $\beta$, IL-6 and cleaved caspase-1. Finally, a TUNEL assay was used to compare the rate of apoptosis of renal tissues among the sham, cecal ligation and puncture (CLP), and CLP + MT groups. The extent of tissue damage in the CLP group was the highest and the extent of tissue damage in the sham group was the lowest. The IHC and western blot analysis showed that the sham group had the highest protein level of PINK1, whereas the CLP group had the lowest protein level of PINK1. By contrast, the sham group had the lowest protein level of NLRP, whereas the CLP group had the highest level of NLRP3. Furthermore, CLP treatment enhanced the protein expression of ASC1 and cleaved caspase-1, whereas the administration of MT reduced the protein expression of $\mathrm{ASC} 1$ and cleaved caspase- 1 to a
\end{abstract}

Correspondence to: Professor Yijun Deng, Department of Critical Care Medicine, Yancheng City No. 1 People's Hospital, 166 Yulong Road, Yancheng, Jiangsu 224006, P.R. China

E-mail: renalmt@126.com

${ }^{*}$ Contributed equally

Key words: melatonin, PTEN-induced putative kinase 1/Parkin1, renal injury, sepsis, apoptosis certain degree. Finally, the apoptotic rate was found to be the highest in the CLP group and the lowest in the sham group. Taken together, in evaluating the therapeutic effect of MT on sepsis-induced renal injury, the results of the present study showed that MT alleviated sepsis-induced renal injury by regulating the expression of PINK1, Parkin1, NLRP3, ASC and cleaved caspase-1 in rats.

\section{Introduction}

Characterized by diffuse inflammatory responses, sepsis is usually caused by a fungal, viral or bacterial infection. According to the most recent data, sepsis is the 7th and 8th highest contributor to mortality in children aged 1-4 years and adults aged 65-75 years worldwide, respectively. In addition, sepsis is the main contributor to mortality in patients with a critical illness (1). For example, as one of the most common and major complications in patients with sepsis, acute kidney injury (AKI) involves the rapid failure of the kidneys to generate sufficient urine, thus impairing the functions of the kidneys to regulate water and ion balance by filtering the blood. AKI substantially elevates healthcare costs and worsens the prognosis of sepsis. For example, the rate of AKI increases with the increasing seriousness of sepsis, and it is estimated that AKI occurs in $64 \%$ of patients with serious hypotension and sepsis within the first $24 \mathrm{~h}$ of the disease.

A decade ago, the inflammasome was considered a major intracellular signaling node containing a receptor for the recognition of cytosolic patterns. In particular, the inflammasome carries absent in melanoma 2 (AIM2)-like receptors and nucleotide-binding oligomerization domain leucine-rich repeat containing receptors (NLRs). The NLR pyrin domain-containing 3 (NLRP3) inflammasome has been the most extensively investigated NLR inflammasome complex, and it acts as a critical signaling platform regulating the maturation of interleukin (IL)-1 $\beta$ and IL-18, two proinflammatory cytokines in the IL-1 family (2). The stimulation of NLRP3 results in the recruitment of the apoptosis-associated speck-like protein containing a $\mathrm{C}$-terminal caspase recruitment domain (ASC), and leads to the transformation of pro-caspase- 1 into its cleaved type (2). Identified as an inflammatory caspase, caspase- 1 is important for the maturation of IL-18 and IL-1 $\beta$ 
into active cytokines. In addition, caspase-1 mediates pyroptosis through its effect on activation and autocatalysis (3). The roles of IL-18 and IL-1 in renal diseases are better understood, although the significance of the inflammasome in the secretion and activation of IL-18 and IL-1 $\beta$ has only been noted relatively recently. A range of primary kidney conditions are associated with the activation of NLRP3 inflammasomes. Similarly, several systemic conditions involving the kidneys, including hypoxia, chronic kidney disease, sepsis, glomerulonephritis, ischemia-reperfusion (I-R) injury, unilateral ureteral obstruction (UUO), crystal nephropathy and glycerol-induced renal failure (4-11), are associated with activation of the NLRP3 IL-1 $\beta /$ IL-18/inflammasome axis. Caspase-9, a caspase for the initialization of mitochondrial apoptosis, is not necessary for the secretion of IL-1 $\beta$ induced by apoptotic stimuli and is not involved in the activation of canonical NLRP3. Furthermore, the absence of NLRP3, caspase-1 or ASC (encoded by Pycard) substantially delays the cell death triggered by nigericin and ATP, suggesting that these NLRP3 stimuli primarily mediate cell death by pyroptosis in this time frame but do not affect the apoptosis of mitochondria (12). The pyroptosis-induced cell death occurs independently of mitochondria by the ASC recruitment of caspase- 8 , and the apoptosis triggered by NLRP3 stimuli is substantially dependent on the activation of its upstream NLRP3 (12). As a potent antioxidant and a scavenger of free radicals (13), melatonin ( $\mathrm{N}$-acetyl-5-methoxytryptamine; MT) is actively taken up by the mitochondria to maintain its homeostasis. In addition, MT has antiseptic effects, targeting the activation of the innate immune pathway involving nuclear factor (NF)- $\mathrm{B}$ (14-17). It has been demonstrated that MT also reduces the activation of NLRP3 inflammasomes in a number of conditions assessed (18).

It has been shown that MT administration can reduce the activation of inflammasomes during sepsis by suppressing the expression of NLRP3 (19). Furthermore, activated inflammasomes are considered to be responsible for sepsis-induced renal injury (20). In the present study, the therapeutic effect of MT in sepsis-induced renal injury, and its effect on the expression of PTEN-induced putative kinase 1 (PINK1), Parkin1, NLRP3, cleaved caspase-1 and ASC were evaluated in rats.

\section{Materials and methods}

Animals. All experiments were performed according to the protocol approved by the Ethical Committee of Yancheng City No. 1 People's Hospital (Yancheng, China). Obtained from the Laboratory Animal Facility of Chinese Academy of Sciences (Yangzhou, China), all Sprague Dawley rats were 10-12 weeks old with a body mass of 20-30 g. All animals were housed in an animal facility with a $12 / 12 \mathrm{~h}$ light-dark cycle at $25^{\circ}$, and had free access to rodent chow and water.

Cecal ligation and puncture (CLP) model. A CLP model was used to induce sepsis. In brief, equithensin $(1 \mathrm{ml} / \mathrm{kg})$ was used to anesthetize the animals by intraperitoneal perfusion, and the rats were fixed on an aseptic operating table. Their cecum was exposed through a 1-cm midline incision in a sterile environment. Subsequently, the cecum was ligated tightly below the ileocecal valve using a 4-0 silk suture. In the subsequent step, a 22-gauge needle was used to puncture the cecum three times to ensure the same severity of sepsis. Subsequently, the cecum was squeezed gently to excrete a small quantity of stool through the puncture site. In the subsequent step, the cecum was placed back into the peritoneal cavity and the abdominal incision was gently stitched in two layers. Finally, $1 \mathrm{ml}$ saline solution was used to resuscitate the animals via subcutaneous injection.

Experimental protocol. All the animals were divided into three groups: A control group (C), a septic group (S), and a septic group administered with MT $(\mathrm{S}+\mathrm{MT})$. The rats in the $\mathrm{S}+\mathrm{MT}$ group were treated with the following three doses of MT-containing 30\% PEG (each dose, $150 \mathrm{mg} / \mathrm{kg}$ ): The first dose was administered intraperitoneally at $30 \mathrm{~min}$ prior to surgery, the second dose was administered immediately following surgery through subcutaneous injection, and the final dose was administered at $4 \mathrm{~h}$ post-surgery through subcutaneous injection. All animals were sacrificed at $8 \mathrm{~h}$ post-CLP to immediately remove the heart, which was rinsed in cold saline and treated to extract the cytosol and nuclei for histological assessment. In order to avoid diurnal variations, all rats underwent surgery between 8:00 a.m. and 12:00 p.m. The MT dose was selected accordingly to inhibit the expression of inducible nitric oxide synthase, a crucial factor of the innate immune in septic rats undergoing CLP.

RNA isolation and reverse transcription-quantitative polymerase chain reaction ( $R T-q P C R)$ analysis. An MirVana ${ }^{\mathrm{TM}}$ miRNA isolation kit (Roche Diagnostics GmbH, Mannheim, Germany) was used to extract total RNA from tissue samples following the manufacturer's protocol. A High-Capacity cDNA reverse transcription kit (Applied Biosystems; Thermo Fisher Scientific, Inc., Waltham, MA, USA) was used to perform reverse transcription to synthesize cDNA (PINK1 or NLRP3) following the protocol of the manufacturer. An ABI 7500 Real-Time PCR system (Applied Biosystems; Thermo Fisher Scientific, Inc.) was used in conjunction with an miScript SYBR $^{\circledR}$-Green PCR kit (Qiagen GmbH, Hilden, Germany) to measure the expression of PINK1 (forward, 5'-CCAAGC TGGCTAGCGTTTAAACG-3'; reverse, 5'-ATGGTCGAC GGCGCTATTCAG-3') and NLRP3 (forward, 5'-GTTTTC ATTCCTGCACTGCCAGTG-3'; reverse, 5'-CAAAAACCC TTCTGTTTACTCACTC-3'). The reaction was performed using 10X Standard Taq Reaction Buffer (5 $\mu \mathrm{l}), 10$ mM dNTPs (1 $\mu \mathrm{l}), 10 \mu \mathrm{M}$ forward primer $(1 \mu \mathrm{l}), 10 \mu \mathrm{M}$ reverse primer (1 $\mu \mathrm{l})$, template DNA (10 ng), Taq DNA polymerase $(0.25 \mu \mathrm{l}$; cat. no. 600280, Agilent Technologies, Inc., Santa Clara, CA, USA) and nuclease-free water $(50 \mu \mathrm{l})$ as follows: $15 \mathrm{~min}$ of initial activation at $95^{\circ} \mathrm{C}$ and $15 \mathrm{sec}$ of denaturation at $94^{\circ} \mathrm{C}$, followed by $30 \mathrm{sec}$ of annealing at $55^{\circ} \mathrm{C}$ and a final extension for $60 \mathrm{sec}$ at $72^{\circ} \mathrm{C}$. GAPDH (forward, 5'-AGAAGGCTGGGG CTCATTTG-3'; reverse, 5'-AGGGGCCATCCACAGTCT TC-3') was used as an internal control to normalize the expression of PINK1 and NLRP3. Finally, the mRNA expression levels of PINK1 and NLRP3 were calculated using the $2^{-\Delta \Delta C q}$ method (21). All reactions were run three times.

ELISA. IL-1 $\beta$, IL-18 and IL-6 ELISA detection kits (R\&D systems, Inc., Minneapolis, MN, USA) were used to determine the levels of IL-1 $\beta$, IL-18 and IL- 6 following the protocol of the supplier. 
Western blot analysis. Ice-cold phosphate-buffered saline (PBS; Hyclone; GE Healthcare Life Sciences, Logan, UT, USA) was used to wash the homogenized tissues, followed by lysis in a RIPA lysis buffer supplemented with $150 \mathrm{mM} \mathrm{NaCl}, 50 \mathrm{mM}$ Tris- $\mathrm{HCl}$ (pH 7.4), 0.1\% SDS, $1 \%$ NP-40 and protease inhibitor cocktails (Roche Diagnostics $\mathrm{GmbH}$ ). Subsequently, the lysate was centrifuged at $25,155 \mathrm{x} \mathrm{g}$ and $4^{\circ} \mathrm{C}$ for $15 \mathrm{~min}$, and boiled for $10 \mathrm{~min}$ in 2-mercaptoethanol. The Bicinchoninic Acid Assay kit (Thermo Fisher Scientific, Inc.) was used to determine protein concentration, based on the concentration of the standard protein supplied in the kit. The proteins in the lysates were resolved using $10 \%$ sodium dodecyl sulfate-polyacrylamide gel electrophoresis and subsequently eletrotransferred onto nitrocellulose membranes (EMD Millipore, Billerica, MA, USA) by $35 \mu \mathrm{g} /$ lane. Subsequently, the membranes were blocked at room temperature for $90 \mathrm{~min}$ in TBST containing $20 \mathrm{mM}$ Tris- $\mathrm{HCl}(\mathrm{pH} \mathrm{7.5)}$ and $0.1 \%$ Tween-20 containing $10 \%$ dry milk, and washed three times in TBST. Primary rabbit anti-PINK1 (cat. no. ab23707), NLRP3 (cat. no. ab214185), ASC (cat. no. ab18193) and caspase-1 antibodies (cat. no. ab1872, 1:5,000 dilution, Abcam, Cambridge, UK) and mouse anti- $\beta$-actin antibodies (cat. no. ab8226, 1:8,000 dilution, Abcam) were used to incubate the membranes at room temperature for $2 \mathrm{~h}$, followed by three TBST washes. The horseradish peroxidase (HRP)-linked anti-mouse and anti-rabbit IgG secondary antibodies at a dilution of 1:15,000 (cat. nos. AC111P and AQ132P, Sigma-Aldrich; Merck KGaA,Darmstadt, Germany) were used to treat the membrane for $2 \mathrm{~h}$ at room temperature. An ODYSSEY infrared imaging system (LI-COR Biosciences, Lincoln, NE, USA) was used to visualize protein signals. All experiments were performed in triplicate.

Immunohistochemistry(IHC). Kidney samples were harvested, fixed in $4 \%$ paraformaldehyde for $48 \mathrm{~h}$, dehydrated, embedded in paraffin and sectioned (size, $0.4 \mu \mathrm{m}$ ). Gradient ethanol was used to dewax and hydrate the samples, followed by $25 \mathrm{~min}$ of antigen retrieval in $10 \mathrm{mM}$ citrate ( $\mathrm{pH}$ 6.0) under a $720 \mathrm{~W}$ heating condition in a microwave. Subsequently, the sections were cooled at room temperature for $30 \mathrm{~min}$, blocked in $3 \%$ hydrogen peroxide for $15 \mathrm{~min}$, and incubated at room temperature for $2 \mathrm{~h}$ with primary goat anti-PINK1 and NLRP3 (1:500 dilution, Abcam) antibodies. Following sample washing with TBST, the HRP-linked anti-goat IgG secondary antibodies at a dilution of 1:1,500 (Sigma; EMD Millipore) were used to treat the samples for $2 \mathrm{~h}$ at room temperature. A 3,3'-diaminobenzidine substrate kit (Vector Laboratories, Inc., Burlingame, CA, USA) was used to visualize bound antibodies, and hematoxylin was used to stain the cell nuclei.

Hematoxylin and eosin $(H \& E)$ staining. At $48 \mathrm{~h}$ post-CLP, the kidney samples were harvested, fixed in $4 \%$ paraformaldehyde for $48 \mathrm{~h}$, dehydrated, embedded in paraffin and sectioned. H\&E was used to stain the sections, which were then viewed under a light microscope.

TUNEL. The apoptotic cells were examined using TUNEL assays (DeadEnd ${ }^{\mathrm{TM}}$ Fluorometric TUNEL system, Promega Corporation) in accordance with the manufacturer's protocol. Subsequently, TUNEL-positive cells in each tissue samples were determined using HPFs (x400 magnification). Three independent tests were run.
Statistical analysis. All data are presented as the mean \pm standard deviation, and SPSS (version 11.5; SPSS, Inc., Chicago, IL, USA) was used to perform statistical analysis. All results were original and strictly verified for their correctness. Two-way analysis of variance with a Holms-Sidak post hoc test was used to perform the statistical comparisons between groups. $\mathrm{P}<0.05$ was considered to indicate a statistically significant difference.

\section{Results}

Injury of renal tissue differs among treatment groups. The animals were divided into three groups: A sham group, a septic group induced by CLP (CLP group) and a septic group treated with MT (CLP + MT group). The renal tissues were collected from all rats, and H\&E staining was performed to determine the extent of tissue damage among the sham, CLP and CLP + MT groups. As shown in Fig. 1, the extent of tissue damage in the CLP group was the highest and the extent of tissue damage in the sham group was the lowest.

Differential expression of PINK1 and NLRP3 among treatment groups. IHC was performed to examine the protein expression of PINK3 and NLRP3 among the sham, CLP, and CLP + MT groups. As shown in Fig. 2, the protein level of PINK1 was highest in the CLP group, whereas the sham group showed the lowest protein level of PINK1. As shown in Fig. 3, the sham group showed the lowest protein level of NLRP3, whereas he CLP group exhibited the highest protein level of NLRP3.

Apoptosis in the renal tissues among treatment groups. A TUNEL assay was performed to detect the apoptosis of renal cells in the samples from the sham, CLP, and CLP + MT groups. As shown in Fig. 4, CLP treatment increased cell apoptosis in the renal tissues compared with that in the sham group, whereas the administration of MT partially reduced the level of cell apoptosis.

Levels of PINK1, NLRP3, ASC1 and cleaved caspase-1 are altered among treatment groups. Western blot analysis was performed to measure the protein levels of PINK1, NLRP3, ASC1 and cleaved caspase-1 among the sham, CLP, and CLP + MT groups. As shown in Fig. 5, the protein level of PINK1 (Fig. 5A and B) in the sham group was the lowest, whereas the protein level of PINK1 in the CLP group was the highest. By contrast, the levels of ASC1 (Fig. 5A and C), cleaved caspase-1 (Fig. 5A and D) and NLRP3 (Fig. 5A and E) in the sham group were the lowest, whereas the levels of these proteins in the CLP group were the highest. These results suggest that CLP increased the protein levels of NLRP3, ASC1 and cleaved caspase-1, and decreased the protein level of PINK1.

Levels of IL-18,IL-1 $\beta$ and IL-6 among treatment groups. ELISA was used to examine the protein levels of IL-18, IL-1 $\beta$ and IL-6 among the sham, CLP, and CLP + MT groups. As shown in Fig. 6A-C, the sham group showed the lowest protein levels of IL-18, IL-1 $\beta$ and IL-6, whereas the CLP group showed the highest protein levels of IL-18, IL-1 $\beta$ and IL-6, indicating 

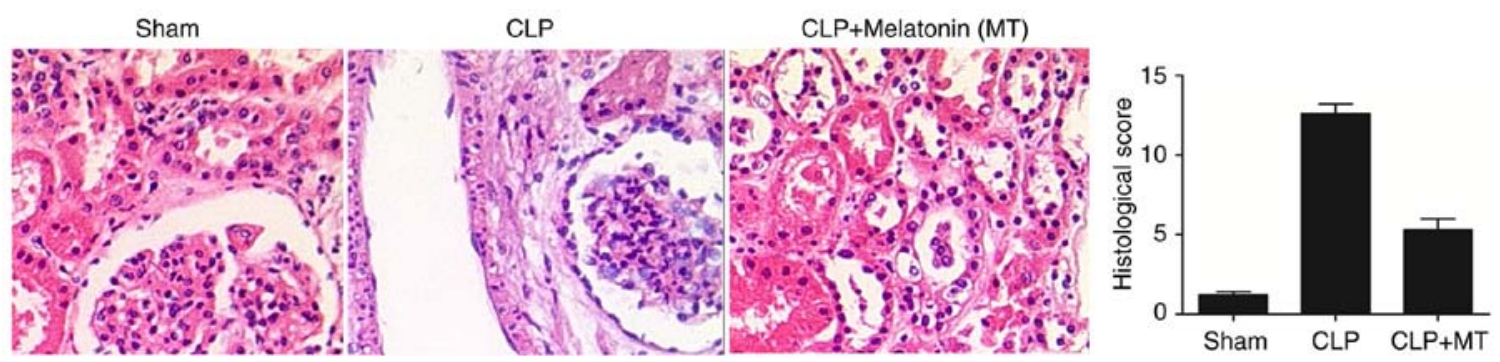

Figure 1. Hematoxylin and eosin staining was performed to detect the extent of renal tissue damage among the sham, CLP and CLP + MT groups (magnification, $\mathrm{x} 40$ ). It was found that CLP treatment increased the extent of tissue damage, which were partially relieved by MT treatment compared with the control. CLP, cecal ligation and puncture; MT, melatonin.
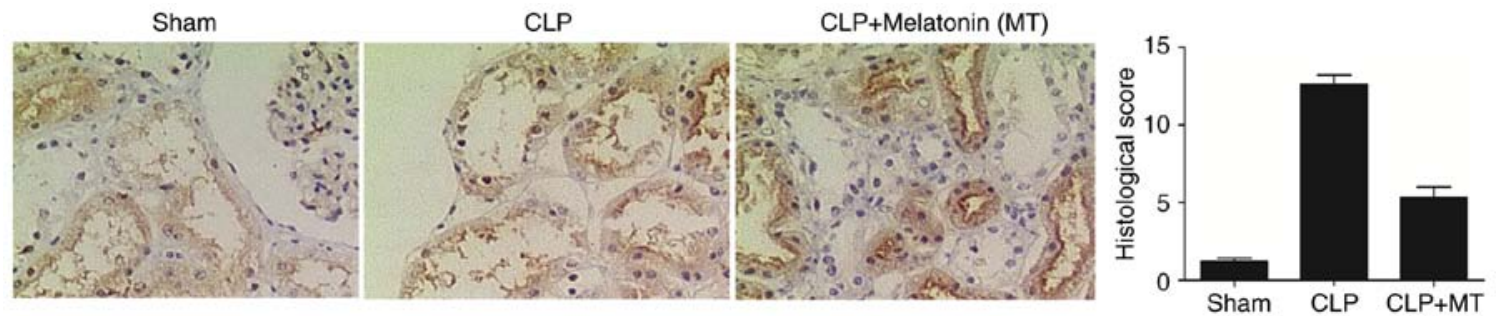

Figure 2. Immunohistochemistry was used to detect the expression of PINK1 among the sham, CLP and CLP + MT groups (magnification, $\mathrm{x} 40$ ). The level of PINK1 was lowest in the CLP group and highest in the sham group compared with the control. CLP, cecal ligation and puncture; MT, melatonin; PINK1, PTEN-induced putative kinase 1.
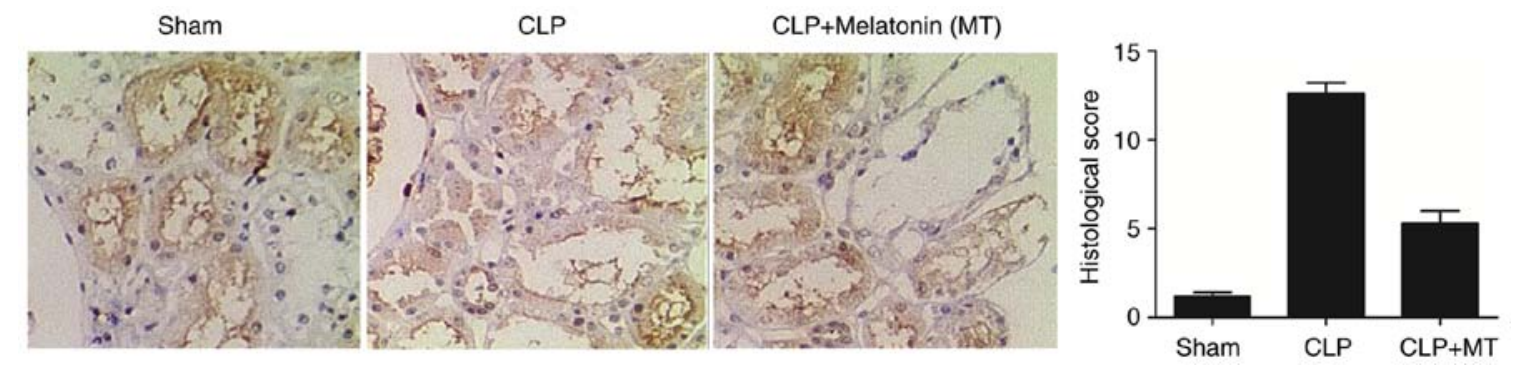

Figure 3. Immunohistochemistry was used to detect the expression of NLRP3 among the sham, CLP and CLP + MT groups (magnification, x40). The levels of NLRP3 was the highest in the CLP group and the lowest in the sham group compared with the control. CLP, cecal ligation and puncture; MT, melatonin; NLRP3, nucleotide-oligomerization binding domain and leucine-rich repeat pyrin domain-containing 3.
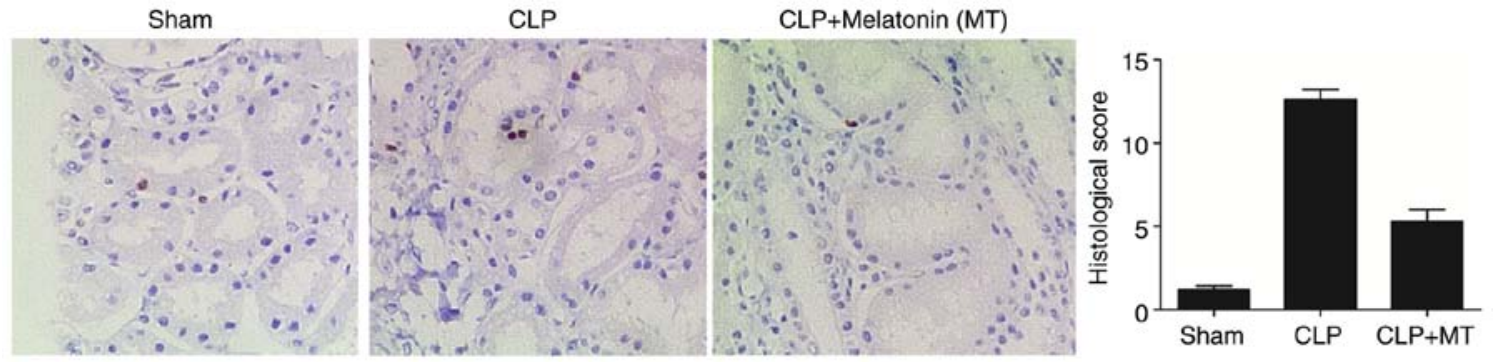

Figure 4. TUNEL assay to examine the apoptosis of renal tissues among the sham, CLP and CLP + MT groups (magnification, $x 40$ ). CLP treatment increased the apoptosis of renal tissues, whereas MT treatment reduced the tissue apoptosis in the CLP group compared with the control. CLP, cecal ligation and puncture; MT, melatonin.

that CLP downregulated the levels of IL-18, IL-1 $\beta$ and IL-6. Superoxide dismutase 2 (SOD2) was downregulated in the CLP group compared with that in the sham group, whereas MT treatment increased the downregulated expression of SOD2 in the CLP group.
Effect of MT treatment on renal functions. In the CLP group, the levels of blood urea nitrogen (BUN) and creatinine $(\mathrm{Cr})$ were substantially increased, whereas treatment with MT partially restored the renal functions, as shown in Fig. 7. 

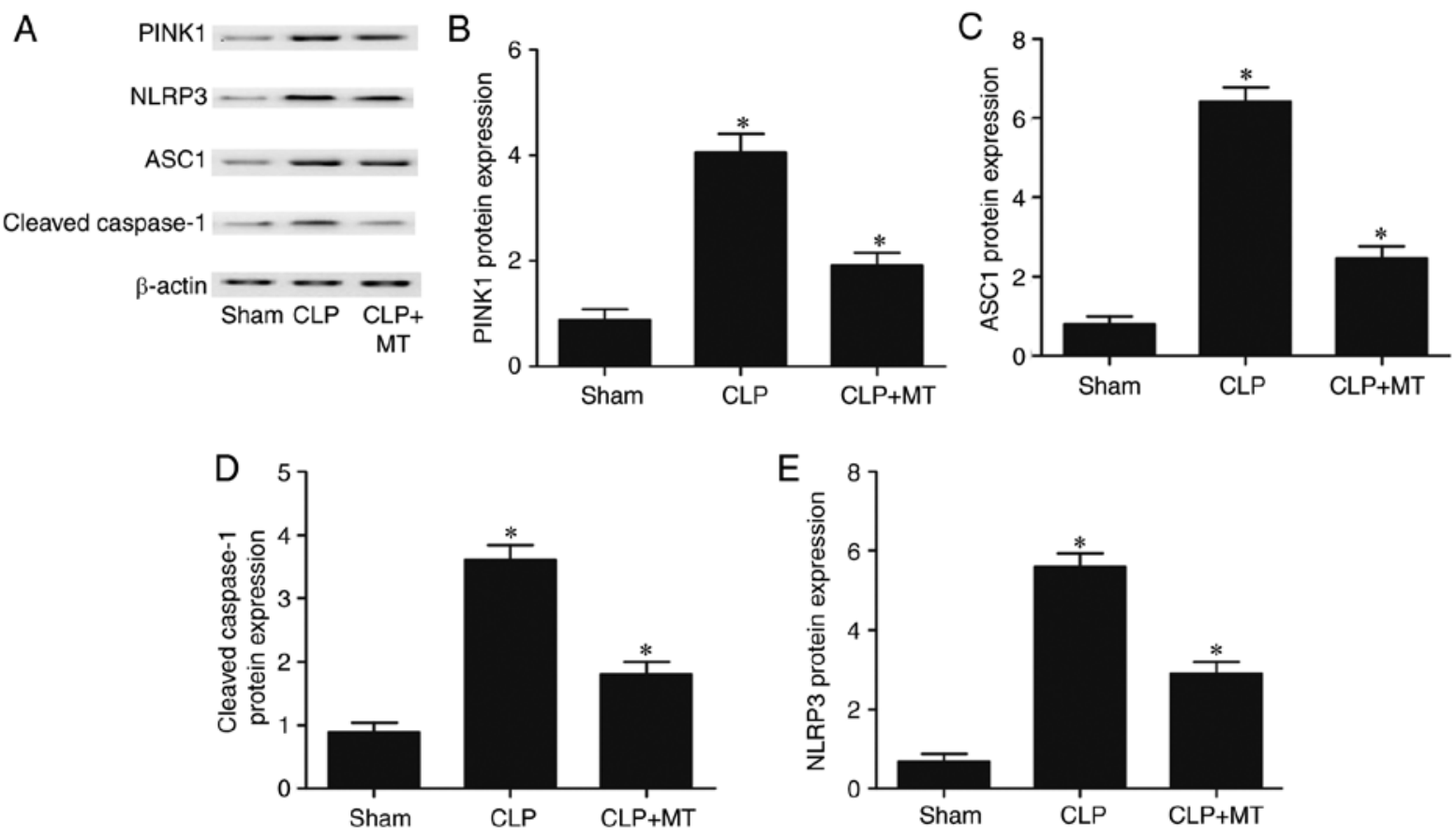

Figure 5. Comparison of protein levels of PINK1, NLRP3, ASC1 and cleaved caspase-1 among the sham, CLP, and CLP + MT groups. Western blot analysis was performed to compare protein levels (one-way analysis of variance, ${ }^{*} \mathrm{P}<0.05$, compared with the control). (A) CLP treatment increased the expression of NLRP3, ASC1, cleaved caspase-1 and PINK1. MT administration showed the opposite trend. (B) CLP treatment upregulated the expression of PINK1, which was partially reduced by MT. (C) Protein level of ASC was highest in the CLP group and lowest in the sham group. (D) Protein level of cleaved caspase-1 was highest in the CLP group and lowest in the sham group. (E) Protein level of NLRP3 was highest in the CLP group and lowest in the sham group. CLP, cecal ligation and puncture; MT, melatonin; PINK1, PTEN-induced putative kinase 1; NLRP3, nucleotide-oligomerization binding domain and leucine-rich repeat pyrin domain-containing 3; ASC1, apoptosis-associated speck-like protein, containing a C-terminal caspase recruitment domain.

A
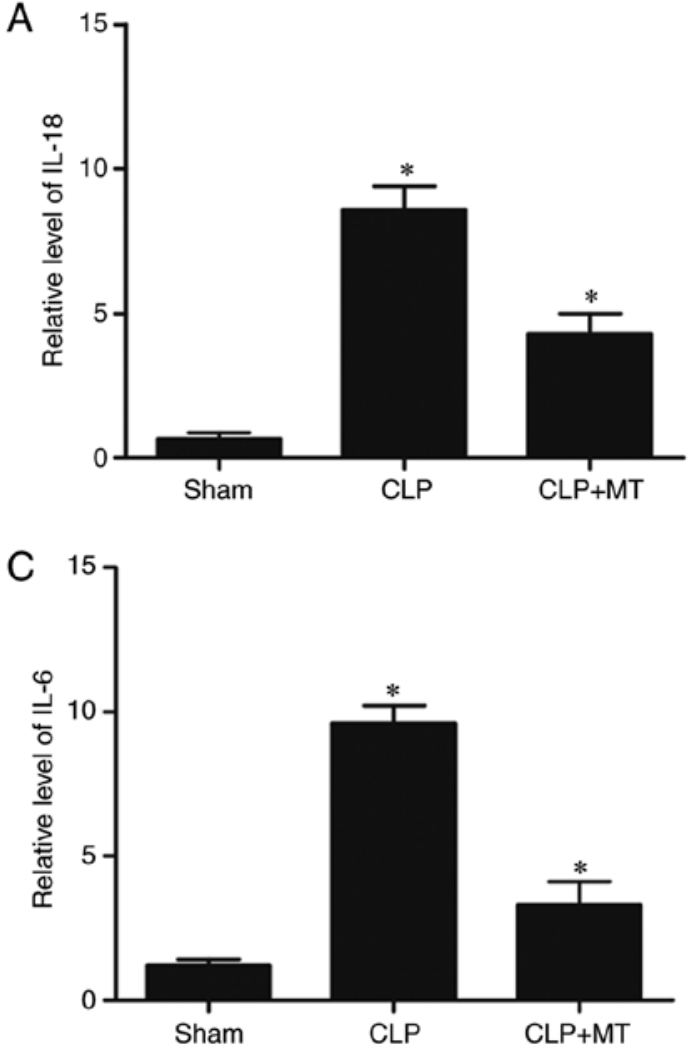

B

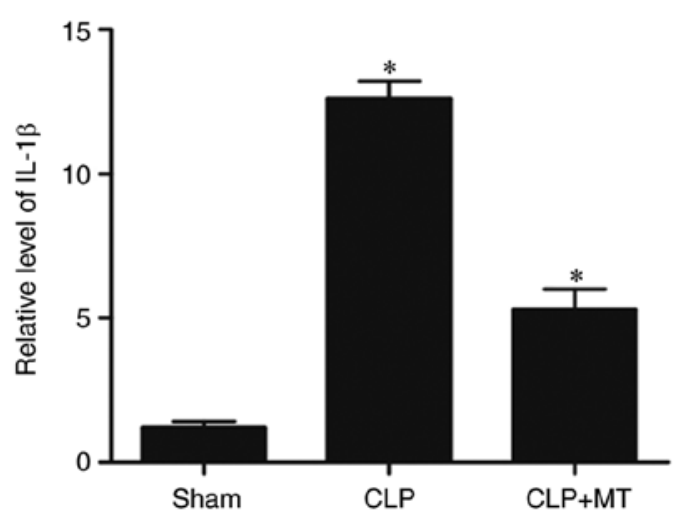

D

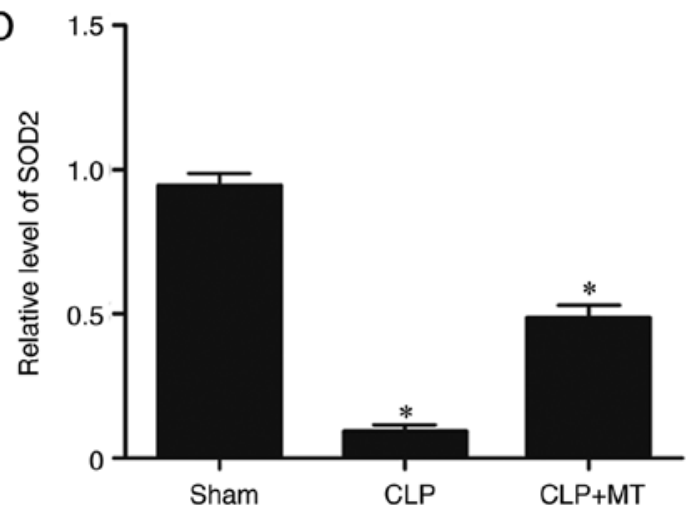

Figure 6. ELISA was used to compare protein levels of IL-18, IL-1 $\beta$ and IL-6 among the sham, CLP, and CLP + MT groups (one-way analysis of variance, ${ }^{*} \mathrm{P}<0.05$, compared with the control). (A) Protein level of IL-18 was highest in the CLP group and lowest in the sham group. (B) Protein level of IL-1 $\beta$ was highest in the CLP group and lowest in the sham group. (C) Protein level of IL-6 was highest in the CLP group and lowest in the sham group. (D) Protein level of SOD2 was lowest in the CLP group and highest in the sham group. CLP, cecal ligation and puncture; MT, melatonin; IL, interleukin; SOD2, superoxide dismutase 2. 


\section{Discussion}

It is well known that patients with sepsis are particularly at risk of AKI-induced death. The pathophysiological role of AKI in sepsis is complex and involves multiple factors, including reactive oxygen species (ROS), chemokines, and pro-inflammatory cytokines (22). Several studies have demonstrated that the dysregulated activation of NLRP3 inflammasomes is associated with the pathogenesis of AKI and sepsis (23). Prior studies have also shown that dysregulated apoptosis is the main pathophysiological mechanism underlying the progression of mild inflammatory conditions to severe inflammatory conditions $(24,25)$.

Inflammasomes modulate the processing of pro-IL-18 and pro-IL-1 $\beta$ by caspase- 1 , and induce inflammatory cell death (pyroptosis) $(26,27)$. The wide range of these activating stimuli suggests that NLRP3-dependent responses are controlled by complex regulatory mechanisms. The generation of ROS and the modification of thioredoxin interacting protein, TXNIP, have been demonstrated to cooperatively 'authorize' NLRP3 inflammasomes to process IL-1 $\beta$ (28). Previous findings have also indicated that caspase 11 and caspase 8 can affect the reaction of NLRP3 inflammasomes to certain 'non-canonical' stimuli derived from pathogens (29).

MT was first isolated in 1958 as a neuro-hormone primarily synthesized and released from the pineal gland (30). MT is an indoleamine synthesized from tryptophan, an essential amino acid (31). MT has been demonstrated to exert anti-inflammatory effects in the skin, intestine, and the lungs; it can also assist in relieving chronic pelvic pain in women with endometriosis (32-34). It has been shown that, acting as an anti-apoptotic and anti-oxidization agent, MT can decrease the kidney damage induced by I-R, UUO, and severe burns (35-37). It has been shown that, by suppressing nucleocytoplasmic translocation, pretreatment with MT can enhance renal regeneration in folic acid-induced AKI, thus providing further insight on the treatment of AKI (38). In the present study, a sepsis rat model was established by CLP treatment, and H\&E staining was performed to detect the extent of renal tissue damage. The results revealed that the extent of tissue damage in the CLP group was the highest, whereas the extent of tissue damage in the sham group was the lowest. Furthermore, IHC and western blot analyses were performed to determine the protein levels of PINK1 and NLRP3 among the sham, CLP, and CLP + MT groups. The results showed that the CLP group was associated with the highest level of PINK1 and the highest level of NLRP3. In addition, TUNEL assays were performed to detect the apoptosis of renal cells in the tissue samples collected from the sham, CLP, and CLP + MT groups. The results revealed that the CLP group exhibited the highest apoptotic rate, whereas the sham group exhibited the lowest apoptotic rate.

Previously, the E3 ubiquitin ligase Parkin and PINK1 were found to trigger mammalian mitophagy. Genetic studies in Drosophila have further revealed that Parkin and PINK1 have regulatory role in mitochondrial integrity (39). Parkin and PINK1 also facilitate the repair of mitochondrial injury by chemical agents, including carbonyl cyanide m-chlorophenylhydrazone (40). The Parkin and PINK1 pathway of mitophagy is also involved in the modulation of mitochondrial actions. For example, mitochondrial depolarization triggers the binding of Parkin/PINK1 to a mitochondrial adaptor protein known as

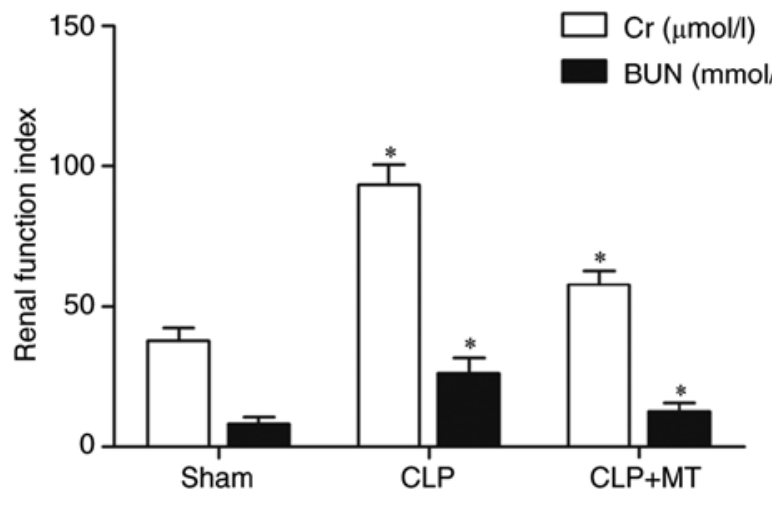

Figure 7. Comparison of renal function indices among treatment groups. Comparison of $\mathrm{Cr}$ among groups. Comparison of BUN among groups (one-way analysis of variance, ${ }^{*} \mathrm{P}<0.05$, compared with the control). CLP, cecal ligation and puncture; MT, melatonin; $\mathrm{Cr}$, creatinine; BUN, blood urea nitrogen.

mitochondria Rho-GTPase, which anchors a kinesin motor complex to the surface of mitochondria (41). Increasing evidence indicates that the interplay of Parkin-NF- $\mathrm{KB}$ counteracts the effect NLRP3-inflammasomes, which can stimulate a wide range of exogenous and endogenous stimuli to result in the caspase 1-triggered maturation of cytokines IL-18 and IL-1 $\beta$. A previous study demonstrated the role of dopamine as an endogenous suppressor of NLRP3 inflammasome stimulation via microglial cells and dopamine receptor signalling (42). NF- $\mathrm{KB}$ not only primed the inflammasomes by mediating the production of pro-IL-1 $\beta$ and NLRP3, but also prevented the excessive activation of NLRP3 by enhancing Parkin-dependent mitophagy. In the present study, ELISA was performed to compare the protein levels of IL-18, IL-1 $\beta$ and IL- 6 among the sham, CLP, and CLP + MT groups. The results showed that the CLP group was associated with the highest protein levels of IL-18, IL-1 $\beta$ and IL-6, whereas the sham group was associated with the lowest protein levels of IL-18, IL-1 $\beta$ and IL-6.

In conclusion, MT-mediated cell apoptosis protects against sepsis-induced renal injury by affecting the PINK1/Parkin1 signaling pathway induced by NLRP3 inflammasomes. The data of the present study showed that MT administration mitigated the activation of inflammasomes in sepsis by suppressing the expression of NLRP3 via inhibiting the PINK1/Parkin1 signaling pathway. The overexpression of NLRP3 promoted the secretion of inflammatory cytokines (IL-1 $\beta$ and IL-18), which promoted cell apoptosis and subsequently induced renal injury in sepsis.

\section{Acknowledgements}

Not applicable.

\section{Funding}

No funding was received.

\section{Availability of data and materials}

The datasets used and/or analyzed during the current study are available from the corresponding author on reasonable request. 


\section{Authors' contributions}

WD, HH and YD designed the study and prepared the manuscript. LS and SH collected the literature, collected the data and prepared the manuscript. LZ and LX analyzed and generated the figures. All authors approved the final manuscript.

\section{Ethics approval and consent to participate}

All experiments were performed according to the protocol approved by the Ethical Committee of Sheyang Zhenyang Hospital (Yancheng, China).

\section{Patient consent for publication}

Not applicable.

\section{Competing interests}

The authors confirm that they have no competing interests.

\section{References}

1. Hotchkiss RS and Karl IE: The pathophysiology and treatment of sepsis. N Engl J Med 348: 138-150, 2003.

2. Davis BK, Wen H and Ting JP: The inflammasome NLRs in immunity, inflammation, and associated diseases. Annu Rev Immunol 29: 707-735, 2011.

3. Lamkanfi M: Emerging inflammasome effector mechanisms. Nat Rev Immunol 11: 213-220, 2011.

4. Edelstein CL, Hoke TS, Somerset H, Fang W, Klein CL, Dinarello CA and Faubel S: Proximal tubules from caspase-1-deficient mice are protected against hypoxia-induced membrane injury. Nephrol Dial Transplant 22: 1052-1061, 2007.

5. Vilaysane A, Chun J, Seamone ME, Wang W, Chin R, Hirota S, Li Y, Clark SA, Tschopp J, Trpkov K, et al: The NLRP3 inflammasome promotes renal inflammation and contributes to CKD J Am Soc Nephrol 21: 1732-1744, 2010.

6. Granfeldt A, Ebdrup L, Tønnesen E and Wogensen L: Renal cytokine profile in an endotoxemic porcine model. Acta Anaesthesiol Scand 52: 614-620, 2008.

7. Chen A, Sheu LF, Chou WY, Tsai SC, Chang DM, Liang SC, Lin FG and Lee WH: Interleukin-1 receptor antagonist modulates the progression of a spontaneously occurring $\operatorname{IgA}$ nephropathy in rats. Am J Kidney Dis 30: 693-702, 1997.

8. Furuichi K, Wada T, Iwata Y, Kokubo S, Hara A, Yamahana J, Sugaya T,Iwakura Y,Matsushima K, Asano M, et al: Interleukin-1dependent sequential chemokine expression and inflammatory cell infiltration in ischemia-reperfusion injury. Crit Care Med 34 2447-2455, 2006.

9. Bani-Hani AH, Leslie JA, Asanuma H, Dinarello CA, Campbell MT, Meldrum DR, Zhang H, Hile K and Meldrum KK IL-18 neutralization ameliorates obstruction-induced epithelial-mesenchymal transition and renal fibrosis. Kidney Int 76 500-511, 2009.

10. Mulay SR, Kulkarni OP, Rupanagudi KV, Migliorini A, Darisipudi MN, Vilaysane A, Muruve D, Shi Y, Munro F, Liapis $\mathrm{H}$ and Anders HJ: Calcium oxalate crystals induce renal inflammation by NLRP3-mediated IL- $1 \beta$ secretion. J Clin Invest 123: 236-246, 2013.

11. Homsi E, Janino P and de Faria JB: Role of caspases on cell death, inflammation, and cell cycle in glycerol-induced acute renal failure. Kidney Int 69: 1385-1392, 2006.

12. Allam R, Lawlor KE, Yu EC, Mildenhall AL, Moujalled DM, Lewis RS, Ke F, Mason KD, White MJ, Stacey KJ, et al: Mitochondrial apoptosis is dispensable for NLRP3 inflammasome activation but non-apoptotic caspase- 8 is required for inflammasome priming. EMBO Rep 15: 982-990, 2014.

13. Galano A, Tan DX and Reiter RJ: On the free radical scavenging activities of melatonin's metabolites, AFMK and AMK. J Pineal Res 54: 245-257, 2013.
14. López A, García JA, Escames G, Venegas C, Ortiz F, López LC and Acuña-Castroviejo D: Melatonin protects the mitochondria from oxidative damage reducing oxygen consumption, membrane potential, and superoxide anion production. J Pineal Res 46: 188-198, 2009.

15. Acuña Castroviejo D, López LC, Escames G, López A, García JA and Reiter RJ: Melatonin-mitochondria interplay in health and disease. Curr Top Med Chem 11: 221-240, 2011.

16. Crespo E, Macías M, Pozo D, Escames G, Martin M, Vives F, Guerrero JM and Acuña Castroviejo D: Melatonin inhibits expression of the inducible NO synthase II in liver and lung and prevents endotoxemia in lipopolysaccharide-induced multiple organ dysfunction syndrome in rats. FASEB J 13: 1537-1546, 1999.

17. Escames G, López LC, Tapias V, Utrilla P, Reiter RJ, Hitos AB, León J, Rodríguez MI and Acuña Castroviejo D: Melatonin counteracts inducible mitochondrial nitric oxide synthase-dependent mitochondrial dysfunction in skeletal muscle of septic rats. J Pineal Res 40: 71-78, 2006.

18. García JA, Volt H, Venegas C, Doerrier C, Escames G, López LC and Acuña Castroviejo D: Disruption of the NF- $\mathrm{B}$ B/NLRP3 connection by melatonin requires retinoid-related orphan receptor- $\alpha$ and blocks the septic response in rats. FASEB J 29: 3863-3875, 2015.

19. Rahim I, Djerdjouri B, Sayed RK, Fernández-Ortiz M, Fernández-Gil B, Hidalgo-Gutiérrez A, López LC, Escames G, Reiter RJ and Acuña-Castroviejo D: Melatonin administration to wild-type rats and nontreated NLRP3 mutant rats share similar inhibition of the inflammatory response during sepsis. J Pineal Res 63, 2017.

20. Patiutko IuI, Khubiev AI, Poliakov MI, Kirsanov MI and Sorokin AI: Functional results of gastrectomy in cancer in relation to the surgical method and creation of esophago-intestinal anastomosis. Vestn Khir Im I I Grek 143: 23-26, 1989 (In Russian).

21. Livak KJ and Schmittgen TD: Analysis of relative gene expression data using real-time quantitative PCR and the 2(-Delta Delta C(T)) method. Methods 25: 402-408, 2001.

22. Schrier RW and Wang W: Acute renal failure and sepsis. N Engl J Med 351: 159-169, 2004.

23. Melnikov VY, Ecder T, Fantuzzi G, Siegmund B, Lucia MS, Dinarello CA, Schrier RW and Edelstein CL: Impaired IL-18 processing protects caspase-1-deficient rats from ischemic acute renal failure. J Clin Invest 107: 1145-1152, 2001.

24. Jimenez MF, Watson RW, Parodo J, Evans D, Foster D, Steinberg M, Rotstein OD and Marshall JC: Dysregulated expression of neutrophil apoptosis in the systemic inflammatory response syndrome. Arch Surg 132: 1263-1269, 1997.

25. Mangan DF, Mergenhagen SE and Wahl SM: Apoptosis in human monocytes: Possible role in chronic inflammatory diseases. J Periodontol 64: 461-466, 1993.

26. Schroder K and Tschopp J: The inflammasomes. Cell 140: 821-832, 2010.

27. Bergsbaken T, Fink SL and Cookson BT: Pyroptosis: Host cell death and inflammation. Nat Rev Microbiol 7: 99-109, 2009.

28. Zhou R, Tardivel A, Thorens B, Choi I and Tschopp J: Thioredoxin-interacting protein links oxidative stress to inflammasome activation. Nat Immunol 11: 136-140, 2010.

29. Gringhuis SI, Kaptein TM, Wevers BA, Theelen B, van der Vlist M, Boekhout T and Geijtenbeek TB: Dectin-1 is an extracellular pathogen sensor for the induction and processing of IL-1 $\beta$ via a noncanonical caspase-8 inflammasome. Nat Immunol 13: 246-254, 2012

30. Tamura H, Nakamura Y, Korkmaz A, Manchester LC, Tan DX, Sugino N and Reiter RJ: Melatonin and the ovary: Physiological and pathophysiological implications. Fertil Steril 92: 328-343, 2009.

31. Reiter RJ, Rosales-Corral SA, Manchester LC and Tan DX: Peripheral reproductive organ health and melatonin: Ready for prime time. Int J Mol Sci 14: 7231-7272, 2013.

32. Siah KT, Wong RK and Ho KY: Melatonin for the treatment of irritable bowel syndrome. World J Gastroenterol 20: 2492-2498, 2014.

33. Schwertner A, Conceição Dos Santos CC, Costa GD, Deitos A, de Souza A, de Souza IC, Torres IL, da Cunha Filho JS and Caumo W: Efficacy of melatonin in the treatment of endometriosis: A phase II, randomized, double-blind, placebo-controlled trial. Pain 154: 874-881, 2013. 
34. de Matos Cavalcante AG, de Bruin PF, de Bruin VM, Nunes DM, Pereira ED, Cavalcante MM and Andrade GM: Melatonin reduces lung oxidative stress in patients with chronic obstructive pulmonary disease: A randomized, double-blind, placebo-controlled study. J Pineal Res 53: 238-244, 2012.

35. Bai XZ, He T, Gao JX, Liu Y, Liu JQ, Han SC, Li Y, Shi JH, Han JT, Tao K, et al: Melatonin prevents acute kidney injury in severely burned rats via the activation of SIRT1. Sci Rep 6: 32199, 2016.

36. Yildirim ME, Badem H, Cakmak M, Yilmaz H, Kosem B, Karatas OF, Bayrak R and Cimentepe E: Melatonin protects kidney against apoptosis induced by acute unilateral ureteral obstruction in rats. Cent European J Urol 69: 225-230, 2016.

37. Chang YC, Hsu SY, Yang CC, Sung PH, Chen YL, Huang TH, Kao GS, Chen SY, Chen KH, Chiang HJ, et al: Enhanced protection against renal ischemia-reperfusion injury with combined melatonin and exendin- 4 in a rodent model. Exp Biol Med (Maywood) 241: 1588-1602, 2016.

38. Zhu F, Chong Lee Shin OL, Xu H, Zhao Z, Pei G, Hu Z, Yang J, Guo Y, Mou J, Sun J, et al: Melatonin promoted renal regeneration in folic acid-induced acute kidney injury via inhibiting nucleocytoplasmic translocation of HMGB1 in tubular epithelial cells. Am J Transl Res 9: 1694-1707, 2017.
39. Greene JC, Whitworth AJ, Kuo I, Andrews LA, Feany MB and Pallanck LJ: Mitochondrial pathology and apoptotic muscle degeneration in Drosophila parkin mutants. Proc Natl Acad Sci USA 100: 4078-4083, 2003

40. Narendra D, Tanaka A, Suen DF and Youle RJ: Parkin is recruited selectively to impaired mitochondria and promotes their autophagy. J Cell Biol 183: 795-803, 2008.

41. Wang X, Winter D, Ashrafi G, Schlehe J, Wong YL, Selkoe D, Rice S, Steen J, LaVoie MJ and Schwarz TL: PINK1 and Parkin target Miro for phosphorylation and degradation to arrest mitochondrial motility. Cell 147: 893-906, 2011.

42. Yan Y, Jiang W, Liu L, Wang X, Ding C, Tian Z and Zhou R: Dopamine controls systemic inflammation through inhibition of NLRP3 inflammasome. Cell 160: 62-73, 2015.

This work is licensed under a Creative Commons Attribution-NonCommercial-NoDerivatives 4.0 International (CC BY-NC-ND 4.0) License. 